\title{
USE OF THE HOSPITAL ANXIETY AND DEPRESSION SCALE (HADS) IN A CARDIAC EMERGENCY ROOM - CHEST PAIN UNIT
}

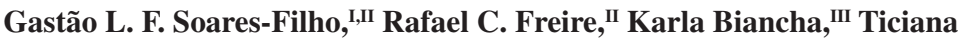

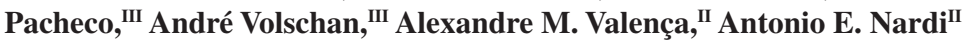 \\ doi: $10.1590 / \mathrm{S1807-59322009000300011}$
}

Soares-Filho GLF, Freire RC, Biancha K, Pacheco T, Volschan A, Valença AM, et al. Use of the hospital anxiety and depression scale (HADS) in a cardiac emergency room - chest pain unit. Clinics. 2009;64(3);209-14.

OBJECTIVE: To determine the prevalence of anxiety and depression in patients complaining of chest pain who seek a chest pain unit attendance.

INTRODUCTION: Patients arriving at a Chest Pain Unit may present psychiatric disorders not identified, isolated or co-morbid to the main illness, which may interfere in the patient prognosis.

METHODOLOGY: Patients were assessed by the "Hospital Anxiety and Depression Scale" as a screening instrument wile following a systematized protocol to rule out the diagnosis of acute coronary syndrome and other potentially fatal diseases. Patients with 8 or more points in the scale were considered "probable case" of anxiety or depression.

RESULTS: According to the protocol, 59 (45.4\%) of 130 patients studied presented Chest Pain of Determined Cause, and 71 (54.6\%) presented Chest Pain of Indefinite Cause. In the former group, in which $43(33.1 \%)$ had acute coronary syndrome, 33.9\% were probable anxiety cases and 30.5\% depression cases. In the second group, formed by patients without acute coronary syndrome or any clinical conditions involving greater morbidity and mortality risk, $53.5 \%$ were probable anxiety cases and $25.4 \%$ depression. CONCLUSION: The high anxiety and depression prevalence observed may indicate the need for early and specialized approach to these disorders. When coronary arterial disease is present, this may decrease complications and shorten hospital stay. When psychiatric disorder appears isolated, is possible to reduce unnecessary repeated visits to emergency room and increase patient's quality of life.

KEYWORDS: Emergency Room; Anxiety; Depression; Chest Pain; Coronary Artery Disease.

\section{INTRODUCTION}

Patients who arrive at the emergency room complaining of chest pain (CP) may present symptoms associated with psychiatric disorders, including anxiety and depression. ${ }^{1}$ Upon assessing patients who presented atypical CP, Wulsin et al. ${ }^{2}$ found that the two most common diagnoses included

IConsultation-Liaison Psychiatry, Hospital Pró-Cardíaco - Rio de Janeiro/ RJ, Brazil.

IInstitute of Psychiatry of the Federal University of Rio de Janeiro (IPUB), Panic \& Respiration Laboratory - Rio de Janeiro/RJ, Brazil.

IIIEmergency Department, Hospital Pró-Cardíaco - Rio de Janeiro/RJ, Brazil.

Tel.: 55212528.1442

Email: galufo@gmail.com

Received for publication on September 15, 2008

Accepted for publication on December 03, 2008 mood disorders and anxiety. A review study ${ }^{3}$ demonstrated that $30.1 \%$ of patients complaining of chest pain who arrived at the emergency room (ER) were diagnosed with a panic disorder (PD), of which $22.4 \%$ exhibited PD with no coronary artery disease (CAD). Patients with anxiety and depression disorders who were not diagnosed as such and thus were not appropriately treated had a tendency to complain of chronic symptoms and to regularly seek medical attention. In analyzing ER patients with atypical pain, Demiryoguran et al. ${ }^{4}$ observed a high probability of anxiety in $31.2 \%$ of these patients. In this group, $78.3 \%$ of the patients reported having previously received care in the ER for the same symptoms. Additional studies have demonstrated the excessive use of medical resources by certain of these patients ${ }^{5}$ who were characterized as having high anxiety and depression scores and low quality of life 
indices. ${ }^{6}$ The objective of the study described herein is to use a self-reporting measure to estimate the prevalence of anxiety and depression in patients admitted to chest pain units.

\section{METHODS}

Among the several instruments designed for psychiatric diagnoses, the Hospital Anxiety and Depression Scale (HADS) distinguishes itself from all other scales due to its ability to assess anxiety and depression without investigating somatic symptoms. HADS is often used to analyze a variety of diseases in the clinical setting. ${ }^{7}$ This method was developed in 1983 and consists of a series of 14 questions, 7 of which are related to anxiety (HAD-A) while the other 7 questions are related to depression (HAD-D). The creators ${ }^{8}$ of the scale considered a score of less than 8 to indicate the lack of any mental disorder, a score equal to or greater than 8 to indicate that a disorder was "probably" present, while a score above 10 was considered to indicate that a patient was "highly likely" to have a disorder. Validation of the Portuguese version, ${ }^{9}$ using the 8 to 9 transition as a cut-off point for each subscale demonstrated a $93.7 \%$ sensitivity for HAD-A and a $84.6 \%$ sensitivity for HAD-D in addition to a $72.6 \%$ specificity for HAD-A and a $90.3 \%$ specificity for HAD-D. The HADS has been used to help diagnose patients suffering from chest pain, ${ }^{10,11}$ atypical chest pain ${ }^{12}$ and heart disease. ${ }^{13}$ It has also been validated in non-cardiac patients complaining of chest pain. ${ }^{14}$

The HADS was administered to chest pain (CP) patients admitted to the Chest Pain Unit (CPU) of a private hospital in Rio de Janeiro from May to August 2006. According to the unit stratification protocol, patients were referred to different acute coronary syndrome (ACS) investigation tracks according to the intensity of chest pain presented on arrival, classified as "definite angina" (Type A), "probable angina" (Type B), "probable non-angina" (Type C) and "definite non-angina" (Type D). Subsequently, the cardiac markers of patients were characterized. Upon admission and every $3 \mathrm{~h}$ thereafter, the serum creatine kinase-MB (CKMB) mass levels were measured in addition to the levels of serum troponin-I, which were analyzed upon admission and at $9 \mathrm{~h}$ post-admission. In addition, an 18-lead electrocardiogram was acquired upon admission and a 12-lead version was reacquired every $3 \mathrm{~h}$ thereafter. Moreover, we recorded a two-dimensional echocardiogram for most patients. In cases where no myocardial necrosis or rest ischemia was detected, a stress test using either a treadmill electrocardiogram or a single-photon emission computed tomographic myocardial scintigraphy was subsequently performed.

We excluded patients with severe clinical conditions, severe respiratory failure, hemodynamic instability and neurological conditions with cognitive involvement. In addition, patients were excluded if diagnosed with dementia, delirium or any psychiatric disorder that causes changes in awareness or in formal thought processes.

The HADS was administered by a nurse or physician who was on call at the CPU after patients had signed an informed consent form, during the waiting time between a blood test and its results or before a diagnostic procedure. If a patient presented a limitation in completing the HADS, such as difficulty reading, the health professional would offer assistance. The health professionals encouraged the patients to choose answers based on symptoms they had experienced during the previous week and asked them to provide spontaneous answers without excessive reflection.

The cut-off value used in this study was 8 for "probable" anxiety or depression. Every patient who presented a score greater than or equal to 8 in the "anxiety" or "depression" subscores of the HADS was referred to a psychiatrist at the hospital. When the diagnosis was subsequently confirmed, a specific treatment regimen was initiated in the CPU.

The current study was approved by the Ethics Committee consistent with the terms of the Helsinki Declaration. A bidirectional model was used for statistical analysis with a $5 \%$ level of statistical significance. SPSS for Windows version 13.0 was used for all analyses.

\section{RESULTS}

A total of 167 questionnaires were administered in this study and 37 potential recruits were excluded for a variety of reasons, including mistakes in filling the questionnaire, refusal to participate, transfer to another institution or inconclusive results. The 130 patients studied included both men $(58.5 \%)$ and women $(41.5 \%)$ between the ages of 31 to 87 years with a mean age of $61.2 \pm 12.1$ years. The most frequent types of reported chest pain were Type B (38.5\%) and Type C (49.2\%) while Type A and Type D were reported significantly less frequently $(10.8 \%$ and $1.5 \%$ of the cases, respectively). Overall, we observed higher than expected anxiety and depression scores upon administering the HADS method, as indicated by the mean scores of $7.33 \pm 4.36$ and $4.78 \pm 3.92$, respectively. A consistent predominance of probable anxiety cases was also observed $-44.6 \%$ of all cases were scored greater than or equal to 8 , while $27.7 \%$ of the cases were determined to be likely depression sufferers with scores greater than or equal to 8 . Of the 130 patients studied, 43 patients (33.1\%) had acute coronary syndrome (ACS). In the latter group, a likely diagnosis of anxiety or depression was recorded in $34.8 \%$ and $27.9 \%$ of all cases, respectively. Interestingly, of all of the patients who reported symptoms of stratified chest pain, 87 (66.9\%) were 
not diagnosed with acute coronary syndrome (NACS). In this group of 87 NACS patients, 16 (18.4\%) patients were diagnosed with a non-coronary cause of chest pain, such as that resulting from pleuritis or pneumonia. However, in 71 of the NACS patients (54.6\%), no cause for the chest pain was discovered prior to discharge from the CPU after investigating and dismissing clinical conditions that evolve with a greater risk of morbidity and mortality (Table 1). Thus we divide the population of 130 patients studied herein into two groups. The first group is composed of 59 patients $(45.4 \%)$ who were diagnosed as having chest pain of a determined cause (PDC) while the second group of 71 patients (54.6\%) were diagnosed as having chest pain of an indetermined cause (PIC).

The PDC group was composed of 43 ACS cases (72.9\%) and 16 NACS cases $(27.1 \%)$ under the given clinical diagnoses. Among the clinically diagnosed NACS cases, $10.2 \%$ of the cases were characterized as coronary artery disease (CAD) not acute, $5 \%$ were patients who had been diagnosed with anxiety and $3.4 \%$ suffered from pleuritic pain. Other diagnoses included pneumonia, costochondritis, lymphatic fistula, exogenous intoxication and tonsillitis, each of which had an incidence of $1.7 \%$. The mean age of this group, which consisted of $64.4 \%$ men and $35.6 \%$ women, was $60.4 \pm 13.6$ years . Type A pain, Type B pain, Type C pain and Type D pain was reported in $23.7 \%, 42.4 \%, 32.2 \%$ and $1.7 \%$ of the cases, respectively. The mean anxiety score as assessed by the HADS method for this group was 6.29 \pm 3.96 points while the depression score was $4.86 \pm 4.07$ points. Thus, $33.9 \%$ of the patients were characterized as likely suffering from anxiety while $30.5 \%$ of the cases were characterized as likely suffering from depression.
Table 1 - Demographic and clinical characteristics

\begin{tabular}{lccc}
\hline & & mean / & SD / \% \\
\hline Age & M & 61.2 & 13.2 \\
Gender & A & 76 & $58.5 \%$ \\
Pain type & B & 14 & $10.8 \%$ \\
& C & 50 & $38.5 \%$ \\
& D & 64 & $49.2 \%$ \\
Non Acute Coronary Syndrome & 2 & $1.5 \%$ \\
Acute Coronary Syndrome & 87 & $66.9 \%$ \\
Chest Pain of Indeterminate Cause & 73 & $33.1 \%$ \\
Chest Pain of Determined Cause & 59 & $54.6 \%$ \\
HADS-A & 7.33 & $45.4 \%$ \\
HADS-D & 4.78 & 4.36 \\
Anxiety & 58 & 3.92 \\
Depression & 36 & $44.6 \%$ \\
\hline
\end{tabular}

The aforementioned PIC group, which also consisted of several NACS cases with no clinical diagnosis, was composed of $53.5 \%$ men and $46.5 \%$ women with a mean age of $61.9 \pm 12.8$ years,. In this group, there were no reports of definite angina-related chest pain (Type A pain). The most common pain classification was type $\mathrm{C}$, which was reported in $63.4 \%$ of the cases, followed by Type B pain (35.2\%) and Type D pain (1.4\%). In this group, the mean anxiety score was $8.20 \pm 4.50$ points and the mean depression score was $4.70 \pm 3.82$ points. Overall, $53.5 \%$ of the patients were characterized as likely suffering from anxiety while $25.4 \%$ were characterized as likely suffering from depression.

Table 2 - Chest Pain of Indeterminate Cause and Chest Pain of Determined Cause - Group comparisons

\begin{tabular}{|c|c|c|c|c|c|}
\hline & & \multicolumn{2}{|c|}{ PIC } & \multicolumn{2}{|c|}{ PDC } \\
\hline & & Mean / n & $\mathrm{SD} / \%$ & Mean / n & $\mathrm{SD} / \%$ \\
\hline Age & & 61.9 & 12.8 & 60.4 & 13.6 \\
\hline \multirow[t]{2}{*}{ Gender } & $\mathrm{F}$ & 33 & $46.5 \%$ & 21 & $35.6 \%$ \\
\hline & M & 38 & $53.5 \%$ & 38 & $64.4 \%$ \\
\hline \multirow[t]{4}{*}{ Pain type (a) } & A & 0 & $0 \%$ & 14 & $23.7 \%$ \\
\hline & B & 25 & $35.2 \%$ & 25 & $42.4 \%$ \\
\hline & $\mathrm{C}$ & 45 & $63.4 \%$ & 19 & $32.2 \%$ \\
\hline & $\mathrm{D}$ & 1 & $1.4 \%$ & 1 & $1.7 \%$ \\
\hline HADS-A (b) & & 8.20 & 4.50 & 6.29 & 3.96 \\
\hline HADS-D & & 4.70 & 3.82 & 4.86 & 4.07 \\
\hline Anxiety (c) & & 38 & $53.5 \%$ & 20 & $33.9 \%$ \\
\hline Depression & & 18 & $25.4 \%$ & 18 & $30.5 \%$ \\
\hline
\end{tabular}

(a) chi-square: 23.656, $3 \mathrm{df}, \mathrm{p}<0.001$; (b) t test $\mathrm{p}<0.011$; (c) chi-square: 5.021, $1 \mathrm{df}$, p: 0.025 . 
In comparing the data gleaned from the PDC and PIC groups (Table 2), our statistical analysis indicates similarities between the demographic data but differences regarding the types of pain and the identified HADS scores. There was no statistically significant difference in terms of the mean age of the patients in the two groups $(p=0.768)$. In regard to gender, while there was a greater proportion of women in the PIC group, this difference did not achieve statistical significance $(p=0.210)$. Interestingly, the PDC group was composed of a significantly higher percentage of patients reporting Type A and Type B pain as compared with the PIC group, which was shown to have a higher percentage of patients reporting Type $\mathrm{C}$ pain (chi-square $=23.656 ; 3$ df; $p<0.001)$. In addition, the mean anxiety scores were significantly higher in the PIC group $(\mathrm{p}=0.011)$, however, this statistically significant difference was not observed for the mean depression scores $(\mathrm{p}=0.819)$. Using the HADS value of 8 as a cut-off point, we observed a significantly higher incidence of anxiety in the PIC group (chi-square = $5.021,1 \mathrm{df}, \mathrm{p}=0.025$ ) while the prevalence of depression was similar between the two groups (chi-square $=0.428,1$ $\mathrm{df}, \mathrm{p}=0.513$ ).

\section{DISCUSSION}

Our key result is the observation that among people who go to an emergency room (ER) complaining of chest pain, which was equivalent to $54.6 \%(71 / 130)$ of the patients in our study, patients belonging to the PIC group would have been discharged with a diagnosis of "no acute coronary syndrome or any other high risk disease" if the HADS method had not been administered. Among those cases, $53.5 \%$ were diagnosed as patients with a probable anxiety disorder while $25.3 \%$ were diagnosed as patients probably suffering from depression. With regard to the presence of anxiety, in comparison with the PDC group, the mean anxiety score was higher in the PIC group. A higher incidence of anxiety was also found in this group using a HADS cut-off point of eight.

Despite the known limitation of this scale as a diagnostic method for research ${ }^{15}$ the application of this very simple test allowed for a significant improvement in the quality of care administered in the ER. Early diagnosis of anxiety or depression disorders ultimately decreased the frequency of ER visits, as well as the cost of treating these patients. ${ }^{16}$ On the other hand, the fact that patients are aware that their chest pain is "not due to a heart disease" has very little impact on the evolution of anxiety disorders. Clinical investigations ${ }^{17}$ even after coronary angiography ${ }^{18}$ are generally unable to prevent new episodes of noncoronary chest pain.
Although a structured interview was not used to confirm the psychiatric diagnoses, in all at-risk cases the mental health care department was contacted and when indicated, the necessary treatment was administered in the CPU. In daily clinical practice, this scale was shown to be useful. A study among hospitalized cardiac patients ${ }^{19}$ found that identifying patients at risk for depression did not require a formal diagnostic tool, but could be achieved using the HADS. Moreover, the sensitivity and specificity of HADS have are already been well established. ${ }^{20} \mathrm{~A}$ review of 747 studies using the $\mathrm{HADS}^{21}$ demonstrated a sufficient ability to assess the symptomatic severity and caseness of anxiety disorders and depression in somatic patients, psychiatric patients, primary care patients and in the general population. However, using the HADS provides higher estimates of depression incidence than the estimates obtained using a diagnostic test such as the PRIME-MD. ${ }^{22}$

Without a screening tool, the ability of ER staff to diagnose anxiety and depression is very limited. In regard to the NACS patients from the PDC group with clinical cause, we note that only $5 \%$ of the patients were diagnosed with anxiety. This observation is consistent with observations reported by other authors. ${ }^{23-25}$ In a study involving ER patients who had reported chest pain, Fleet et al. ${ }^{23}$ noticed that PD was identified in only $2 \%$ of the 108 cases examined. In a separate study, ${ }^{24}$ the diagnosis of a psychiatric disorder was made in only 1 out of 30 patients, demonstrating failure in $97 \%$ of cases. Using a self-reporting measure such as the HADS, it was possible to classify $33.9 \%$ of the patients from the PDC group as likely anxiety sufferers and $30.5 \%$ of patients from this group as probable depression cases. In addition, after observing patients during the first week following acute myocardial infarct, Martin et al. ${ }^{25}$ observed a HADS score greater than 8 for HAD-A questions and for HAD-D questions in $30.4 \%$ and $15.2 \%$ of the cases studied, respectively. The hazardous impact of anxiety and depression on the development of CAD, ${ }^{26-29}$ the effects on the prognosis of the psychiatric disease itself, ${ }^{30}$ and the excessive use of medical resources ${ }^{31}$ have been well established in the literature. Recently it was demonstrated that anxiety and depression predict more problematic adverse cardiac events in patients with stable CAD. ${ }^{32}$ Given the elevated rate of morbidity and mortality associated with depression among cardiac patients, ${ }^{33}$ the HADS may serve as a useful tool in identifying at-risk patients. ${ }^{19}$

\section{CONCLUSIONS}

A significant proportion of patients complaining of symptoms indicative of chest pain who were admitted to the emergency department at our facility had a diagnosable 
psychiatric illness. The HADS method is an easily applied screening method that can be incorporated into daily clinical practice at the ER to allow for the early diagnosis of anxiety and depression disorders in chest pain patients.

\section{REFERENCES}

1. Yingling KW, Wulsin LR, Arnold LM, Rouan GW. Estimated prevalence of panic disorder and depression among consecutive patients seen in an emergency department with acute chest pain. J Gen Intern Med. 1993;8:231-5.

2. Wulsin LR, Arnold LM, Hillard JR. Axis I disorders in ER patients with atypical chest pain. Int J Psychiatry Med. 1991;21:37-46.

3. Lynch P, Galbraith KM. Panic in the emergency room. Can J Psychiatry. 2003;48:361-6.

4. Demiryoguran NS, Karcioglu O, Topacoglu H, Kiyan S, Ozbay D, Onur $\mathrm{E}$, et al. Anxiety disorder in patients with non-specific chest pain in the emergency setting. Emerg Med J. 2006;23:99-102.

5. Coley KC, Saul MI, Seybert AL. Economic burden of not recognizing panic disorder in the emergency department. J Emerg Med. 2009 Jan;36(1):3-7. Epub 2007 Oct 15.

6. Bringager CB, Arnesen H, Friis S, Husebye T, Dammen T. A Long-Term Follow-Up Study of Chest Pain Patients: Effect of Panic Disorder on Mortality, Morbidity, and Quality of Life. Cardiology. 2007;110:8-14.

7. Herrmann C. International experiences with the Hospital Anxiety and Depression Scale--a review of validation data and clinical results. J Psychosom Res. 1997;42:17-41.

8. Zigmond AS, Snaith RP. The hospital anxiety and depression scale. Acta Psychiatr Scand. 1983;67:361-70.

9. Botega NJ, Bio MR, Zomignani MA, Garcia C Jr, Pereira WA. Mood disorders among inpatients in ambulatory and validation of the anxiety and depression scale HAD. Rev Saúde Pública. 1995;29:355-63.

10. Bass C, Chambers JB, Kiff P, Cooper D, Gardner WN. Panic anxiety and hyperventilation in patients with chest pain: a controlled study. Q J Med. 1988; 69:949-59.

11. Goodacre S, Nicholl J. A randomized controlled trial to measure the effect of chest pain unit care upon anxiety, depression, and health-related quality of life. Health Qual Life Outcomes. 2004;2:39.

12. Kuijpers PM, Denollet J, Wellens HJ, Crijns HM, Honig A. Noncardiac chest pain in the emergency department: the role of cardiac history, anxiety or depression and Type D personality. Eur J Cardiovasc Prev Rehabil. 2007;14: 273-9.

13. Bambauer KZ, Locke SE, Aupont O, Mullan MG, McLaughlin TJ. Using the Hospital Anxiety and Depression Scale to screen for depression in cardiac patients. Gen Hosp Psychiatry. 2005; 27:275-84.

14. Kuijpers PM, Denollet J, Lousberg R, Wellens HJ, Crijns H, Honig A. Validity of the hospital anxiety and depression scale for use with patients with non-cardiac chest pain. Psychosomatics. 2003;44:329-35.

15. Silverstone PH. Poor efficacy of the Hospital Anxiety and Depression Scale in the diagnosis of major depressive disorder in both medical and psychiatric patients. J Psychosom Res. 1994;38:441-50.
16. Roy-Byrne PP, Clary CM, Miceli RJ, Colucci SV, Yikang X, Grudzinski AN. The effect of selective serotonin reuptake inhibitor treatment of panic disorder on emergency room and laboratory resource utilization. J Clin Psychiatry. 2001;62:678-82.

17. Dumville JC, MacPherson H, Griffith K, Miles JN, Lewin RJ. Noncardiac chest pain: a retrospective cohort study of patients who attended a Rapid Access Chest Pain Clinic. Fam Pract. 2007;24:152-7.

18. Bass C, Wade C, Hand D, Jackson G. Patients with angina with normal and near normal coronary arteries: clinical and psychosocial state 12 months after angiography. Br Med J. 1983;287:1505-8.

19. Herrmann C, Brand-Driehorst S, Kaminsky B, Leibing E, Staats H, Rüger U., Diagnostic groups and depressed mood as predictors of 22-month mortality in medical inpatients. Psychosom Med. 1998;60:570-7.

20. Strik JJ, Honig A, Lousberg R, Denollet J. Sensitivity and specificity of observer and self-report questionnaires in major and minor depression following myocardial infarction. Psychosomatics. 2001;42:423-8.

21. Bjelland IA, Dahl A, Haug TT, Neckelmann D. The validity of the Hospital Anxiety and Depression Scale. An updated literature review. J Psychosom. Res. 2002;52:69-77.

22. Boyd JH, Weissman MM, Thompson WD, Myers JK. Screening for depression in a community sample. Understanding the discrepancies between depression symptom and diagnostic scales. Arch Gen Psychiatry. 1982;39(10):1195-200.

23. Fleet RP, Dupuis G, Marchand A, Kaczorowski J, Burelle D, Arsenault A, et al. Panic disorder in coronary artery disease patients with noncardiac chest pain. J Psychosom Res. 1998;44:81-90.

24. Wulsin LR, Hillard JR, Geier P, Hissa D, Rouan GW. Screening emergency room patients with atypical chest pain for depression and panic disorder. Int J Psychiatry Med. 1988;18:315-23.

25. Martin CR, Lewin RJ, Thompson DR. A confirmatory factor analysis of the Hospital Anxiety and Depression Scale in coronary care patients following acute myocardial infarction. Psychiatry Res. 2003;120:8594.

26. Frasure-Smith N, Lesperance F, Talajic M. Depression following myocardial infarction. Impact on 6-month survival. JAMA. 1993;270:1819-25

27. Carney RM, Blumenthal JA, Freedland KE, Youngblood M, Veith RC, Burg MM, et al. Depression and late mortality after myocardial infarction in the Enhancing Recovery in Coronary Heart Disease (ENRICHD) study. Psychosom Med. 2004;66:466-74.

28. de Jonge P, Honig A, van Melle JP, Schne AH, Kuyper AM, Tulner D. Non-response to treatment for depression following myocardial infarction: association with subsequent cardiac events. Am J Psychiatry. 2007;164: 1371-8. 
29. Bunker SJ, Colquhoun DM, Esler MD, Hickie IB, Hunt D, Jelinek M, et al. "Stress" and coronary heart disease: psychosocial risk factors. Med J Aust 2003;178:272-6.

30. Meincke U, Hoff P. Psychiatric disorders following myocardial infarction. Med Klin. 2006;101:373-7.

31. Chazov EI, Oganov RG, Pogosova GV, Shal'nova SA, Romasenko LV, Deev AD. Clinico-epidemiological program of the study of depression in cardiological practice in patients with arterial hypertension and ischemic heart disease (COORDINATA). Kardiologiia. 2007;47:29-37.
32. Frasure-Smith N, Lespérance F. Depression and anxiety as predictors of 2-year cardiac events in patients with stable coronary artery disease. Arch Gen Psychiatry. 2008;65:62-71.

33. Martin CR, Thompson DR. A psychometric evaluation of the Hospital Anxiety and Depression Scale in coronary care patients following acute myocardial infarction. Psychol Health Med 2000;5:193-201. 